\title{
Frequent procedural errors and structural complications on endodontical treated teeth
}

\author{
Sanziana Adina Scarlatescu', Irina Gheorghiu' ${ }^{2}$, George Nicola ${ }^{3}$, \\ Andrei O. Al Aloul', Paula Perlea' \\ ${ }^{1}$ Department of Endodontics, \\ "Carol Davila" University of Medicine and Pharmacy, Bucharest, Romania \\ ${ }^{2}$ Department of Restorative Dentistry, \\ "Carol Davila" University of Medicine and Pharmacy, Bucharest, Romania \\ ${ }^{3}$ Department of Medical Legal Elements and Malpractice, \\ "Carol Davila" University of Medicine and Pharmacy, Bucharest, Romania
}

\begin{abstract}
The objectives of root canal treatment are to prevent the apical parodontitis, to heal the acute or cronic apical periodontits and to maintain the results, keeping the teeth on the arch in a functional stage. Thus, accuratelly elimination of soft and hard tissues (cleaning and shaping of the root canal system), disinfection and complete, homogeneous and tridimensional filling of the root canal system are very important to successful endodontic treatment. Modern concepts in endodontic treatment rely on technological progress and therefore many cases are successfully fulfilled, but the practitioner may be put in front of procedural erorrs both during and after endodontic treatment. Apical blockage, ledging, zipping, stripping, perforations in the floor of the pulp canal chamber or root canals perforations are the most common errors, but in many clinical situations they can be prevented and even surpassed once happened.
\end{abstract}

Keywords: procedural errors, apical blockage, ledging, zipping

\section{INTRODUCTION}

A modern endodontic treatment involves a correct, accurate preparation of the root canals, an effective disinfection provided by endodontic irrigation and a sealing of the root canal system by a complete, homogeneous and three-dimensional root canal filling. Modern procedures in endodontic treatment are based primarily on technological progress so that optimal results are obtained in a large percentage of cases; however, procedural errors and problems can occur both during and after endodontic treatment, which jeopardize the success of treatment.

Errors that may occur can be classified as follows:

- Access-related errors - when making the access cavity

- Errors during root canal preparation
- Errors when performing root canal filling

- Structural complications that may occur at various periods after completion of endodontic treatment

\section{ACCESS-RELATEDPROCEDURALERRORS}

Incomplete anaesthesia of the tooth - can be compensated by performing a new anaesthesia or by supplementing it with intrapulpal or intraligamentary anaesthesia.

Treating the wrong tooth - extremely upsetting accident which occurs when the causative tooth has not been correctly identified.

Perforation of the dental crown - occurs on the external lateral surfaces of the upper or lower front teeth, due to the ignorance of the anatomical inclination of the roots in relation to the crowns of the teeth, as a result of their insertion into the jaw 
bones. In the case of these teeth the access will be done perpendicular to the surface of the tooth, and after the burr has penetrated 2-3 $\mathrm{mm}$ inside, it will be reoriented to coincide with the long axis of the root, according to its inclination in the jaw bone (1) (fig. 1).

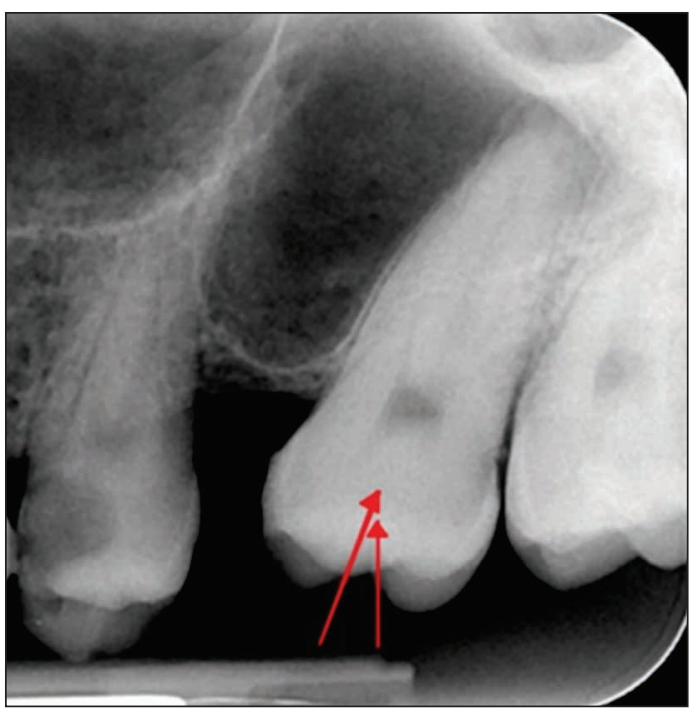

FIGURE 1. Change in the direction of the access burr, after the initial trepanation

Undersized access cavity - provides low visibility, the impossibility of correctly inserting of files, at risk of breaking them during treatment, as well as errors in determining the working length.

Oversized access cavity - weakens the strength of the tooth, leading therefore to the fracture of the coronary walls.

Incomplete removal of the ceiling of the pulp chamber - leads to errors in preparation; at the same time there is an incomplete removal of the pulp, which leads to a subsequent staining and infection of the tooth, as a result of degradation products developed inside the pulp.

Missed root canals - in these cases an acute inflammatory process is observed, even under the conditions of correct treatment and root canal filling. An eccentric X-ray or a CBCT can accurately diagnose the cause.

Perforation of the pulp chamber floor - appears as an extremely unpleasant error in the lateral teeth, especially those with calcifications of the pulp chamber. The main cause is the use of high speed burrs for removing the secondary and tertiary dentine. In these cases, the height of the pulp chamber must be accurately measured on the initial X-ray and low speed burrs or CPR or ProUltra ultrasonic tips should be used with particular care, as not to damage the pulp chamber floor.

Factors on which the successful treatment of the pulp floor chamber perforations depends on:

- the time elapsed since the perforation occurred - the sooner it is treated, the better

- the size of the perforation - perforations less than $3 \mathrm{~mm}$ have a better prognosis. The material of choice is MTA

- location - if the perforation involves the floor chamber, an inflammatory lesion may develop in the furcation. That may be prevented by sealing the perforation as soon as possible. In general, good prognoses have those perforations located at the level of the dental furcation, those located in the middle $1 / 3$ and apical $1 / 3$ of the root canal. A worse prognosis have the perforations located in the upper $1 / 3$ of the root (2) (fig. 2).

- lack of bacterial contamination - the isolation with the rubber dam is very important

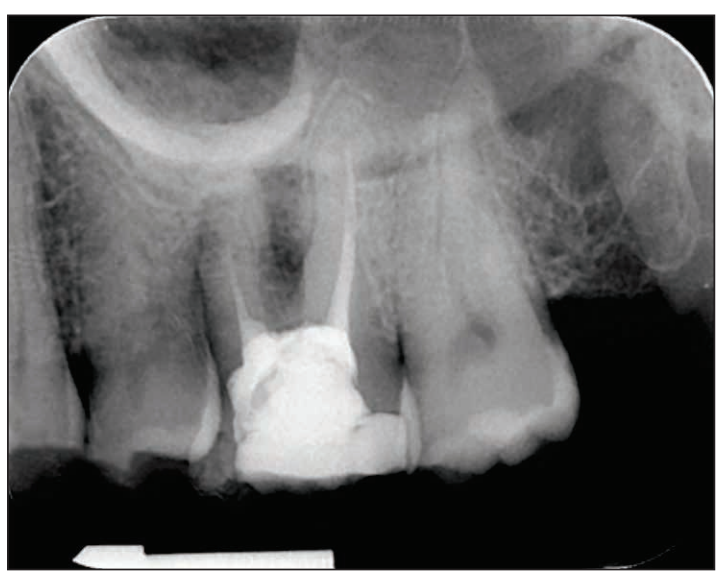

FIGURE 2. Perforation of the pulp floor chamber, lesion developed in the furcation

\section{ERRORS WHICH OCCURS DURING ROOT CANAL PREPARATION}

1. Loss of working length during the treatment

2. Apical blockage

3. Ledge formation

4. Internal apical transposition and zipping, which can be transformed into external apical transposition and root perforation

5. Perforation of the inner wall of the root canal on lateral teeth (stripping)

6. Apical overinstrumentation (loss of apical constriction and enlargement of the apex)

7. Root perforation 
8. Fracture of endodontic instruments in the canal (Separated instruments)

The shortening of the working length arise due to the choice of an inadequate coronary reference point, with undermined dentin cusps, predisposing to fracture throughout treatment appointments.

The apical blockage occurs when tissue debris, dentin chips, dentin mud are compacted at the apical level during the enlargement operations. It contains vital or necrotic pulp remains. In teeth with vital pulp, this error occurs especially when we use the 15 ISO file after the 10 file. 15 ISO file is much thicker than $10 \mathrm{k}$ file and therefore, when the canal is dry, the pulp tissue turns into a hard mass of collagen when pushed with a thick $15 \mathrm{k}$ file. Apical blockage can occur also during retreatment, when at some point the previously patent canal at its full working length, cannot be negotiated anymore, due to remnants of sealers or guttapercha removed from the canal walls.

To prevent this incident, it is recommended to maintain and check the apical patency throughout the treatment, to keep doing recapitulation, as well as to ensure abundant irrigation in the root canal and to clean the files before reinsertion. The use of modern rotary instruments with a special design of the pitch, helical angle and grooves, as to eliminate the debris, can help avoid apical blockage.

Correction is accomplished by using small precurved files (0.8 - 10 ISO), with forward - backwards movements into the axis of the canal, without their rotation (3). Chelating agents and circumferential rotation might also help managing this iatrogenic error.

Canal blockage, decreasing of the working length and incomplete shaping of the endodontic space, is responsible for many failures of endodontic treatment, with flare-ups at different time periods after the end of root canal treatment (fig. 3, 4, 5).

The ledge can result from the repeated pressure application of the same file at a certain level in the root canal. For example, during the filing movements using a rigid file or repeated inserting of a thicker file at a level immediately prior to the beginning of an apical curve and not respecting the order of the instruments. Also using non-precurved stainless-steel files, performing conservative access cavities (Ninja Access), insufficient preflaring, incorrect canal scouting and the absence of a glyde path or incorrect length determination can

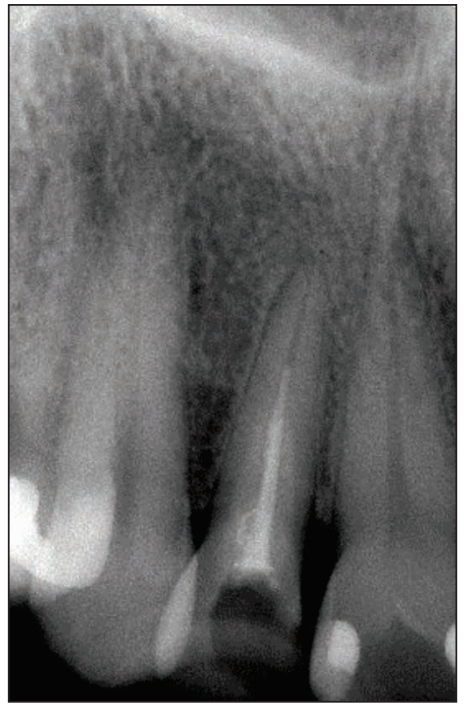

FIGURE 3. Incomplete root canal filling

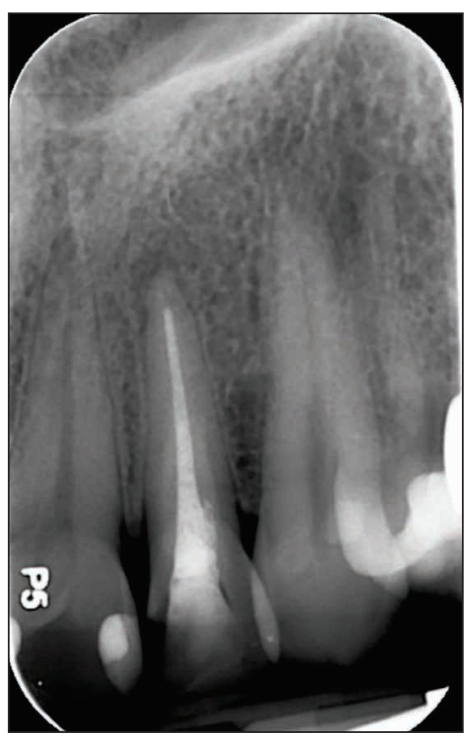

FIGURE 4.

Retreatment, apical blockage, flare-up

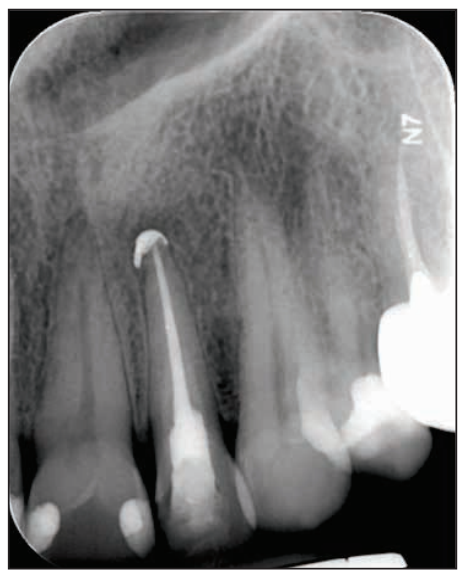

FIGURE 5. Final root canal filling

lead to ledge formation. The favoring factor is obviously the sharp curvature, particularly in narrow or calcified canals, but the determining factor is the incorrect use of files during enlargement and also the alloy and the design of the instruments, especially of the tip and the centering ability in case of rotary instruments. The ledge occurs on the outer side of the canal curvature, not only during 
shaping of the root canal system per primam, but also during retreatment, bypassing a separated instrument or preparation for posts.

The ledge makes the subsequent apical preparation very difficult or impossible. To overcome this iatrogenic error, small 08 or 10 ISO pre-curved hand-files shall be used with 1-2 mm apical slight movements and $30^{\circ}$ watch-winding rotation, in an attempt to resume the initial route of the canal. After bypassing the ledge, gentle push-pull movements of low amplitude helps disband the false route created previously and successively larger instruments can be inserted.

Detecting the ledge in an early stage before the use of larger instruments makes the management of this procedural error easier.

The apical transportation/zipping is caused by straightening the apical third of the canal and shifting the canal path to the outer portion of the curve. By widening the apical portion with large rigid files, the destruction of the initial apical constriction occurs and therefore an irregularly shaped enlarged apical third is formed, like a zipper and an apex with a tear drop or elliptical shape, difficult to clean, disinfect and filled (fig. 6, 7).

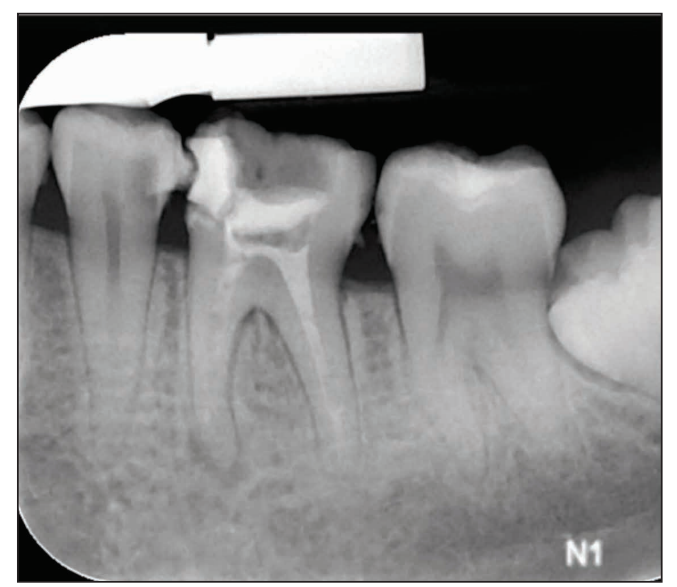

FIGURE 6. Retreatment of 36



FIGURE 7. Zipping and apical transposition
These procedural errors depends on: the shape of the access cavity (small cavities favors it), the alloy from which the files are made (flexible ones have a lower potential to straighten the root canal), the design of the tip of the file (passive vs. cutter), the preparation technique (rotary instrumentation produces less errors), irrigation in the root canal (lack of irrigation favors transpositions), the degree of curvature, as well as the operator's experience (more commonly in beginners) (4), the use of flexible or precurved instruments, without forcing them in curved canals.

Perforation of the internal root wall (stripping) can occur at any time during the preparation of the lateral teeth root canals, mainly in curved canals. Suddenly bleeding in the root canal is an indication of the occurrence of a perforation. Proper use of Gates-Glidden drills or Sx ProTaper instrument with brushing movements on the wall opposite to the furcation during preflaring procedures prevents this error. At the same time, the reduction of the preparation in the coronal and middle part of the canal not only avoids the occurrence of this error, but also improves the mechanical strength of the endodontic treated teeth $(5,6)$.

The instrumentation of a curved canal must take into account its anatomy, should be done using flexible instruments, avoiding the rigid ones and the preparation should be minimal invasive as to be clinically effective. Otherwise, dangerous thinning of the internal wall may occur which, under the masticatory force, may cause cracks in the root wall, with the appearance of communication between the canal and the periodontium, leading to the failure of the treatment and the loss of the tooth (fig. 8, 9).



FIGURE 8. Second lower molar, significant curvature of mesial root 


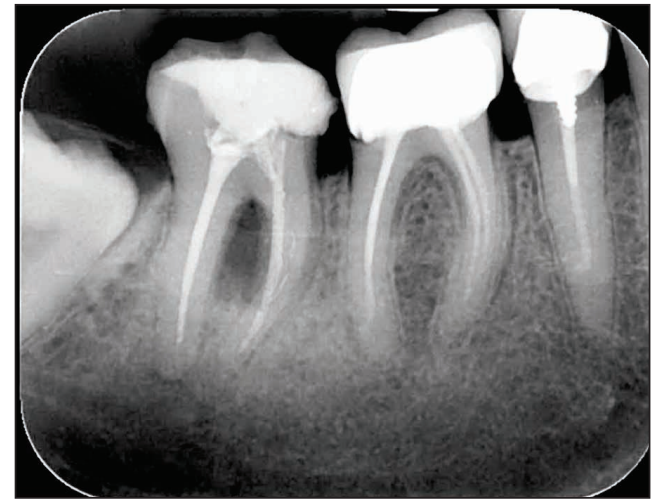

FIGURE 9. Stripping on mesial root

Separated instruments/the fracture of an endodontic instrument is an extremely unpleasant procedural error, caused by the excessive force exerted on the file. The use of endodontic engines with torque-control mechanism is very important for reducing the stress exerted on Ni-Ti instruments. The value of the torque takes into account the beginning and the end of the martensitic clinical transformations that take place in the structure of the files when the canals are instrumented. When the maximum stress value is reached, the engine stops the rotation or rotates the instrument a turn back, in order to prevent deformation or breakage (7). This mechanism protects, but does not provide absolute protection. This is why it is very important to create the smooth glide path first, for the tip of the instrument to be passive and prevent it from breaking.

Using many times rotary instruments in auto-reverse mechanisms leads to their cyclic fatigue and subsequently to their fracture. That's why there are currently single-use systems, with a higher cost price, of course. On the other hand, the process of sterilizing also sometimes affects their structure and decreases the efficiency of the preparation.

The removal of fractured instruments in the canal is also a complicated and expensive process, so this procedural error should be avoided as much as possible (3). Certain files can break even if handled with care. Stress on the instrument can be seen first by the flutes, which may appear "unwound". Instruments with these signs of potential fracture should be discarded.

Small instruments - such as K- 10, 15, even 20 ISO must be carefully examined. They must not be forced into the canal. If a particular instrument does not enter the canal at the desired working length, it will be removed and the tip will be precurved to enter the desired path. In these cases lubricants can also help a lot - Glyde, EDTA.

Factors that prevent the instruments from breaking are: sequential instrumentation, switch to an upper file only after the small one moves freely in the canal, avoid overuse and excessive force, examine the file before use, use flexible instruments made of high quality alloys.

\section{ERRORS IN PERFORMING ROOT CANAL FILLING: OVEREXTENDED ROOT CANAL FILLING}

Overfilling with small amount of sealer (puffs) is in general well tolerated by the body. It is attesting the filling of the entire root canal and in particular the root canal constriction - apical foramen (fig. 10). It is considered that overfilling up to 2 $\mathrm{mm}$ with paste/sealer or gutta-percha cone, without being considered a normality, can be more quickly integrated by the body (8), but massive overextensions should always be avoided by accurately determining the working length, the exact preparation of the root canals, the suitable choice of a master cone and a proper root canal filling technique, avoiding the introduction of large quantity of sealing cement (9) (fig. 11). Overextended fillings endanger relations with neighboring anatomical structures and can only be removed surgically.

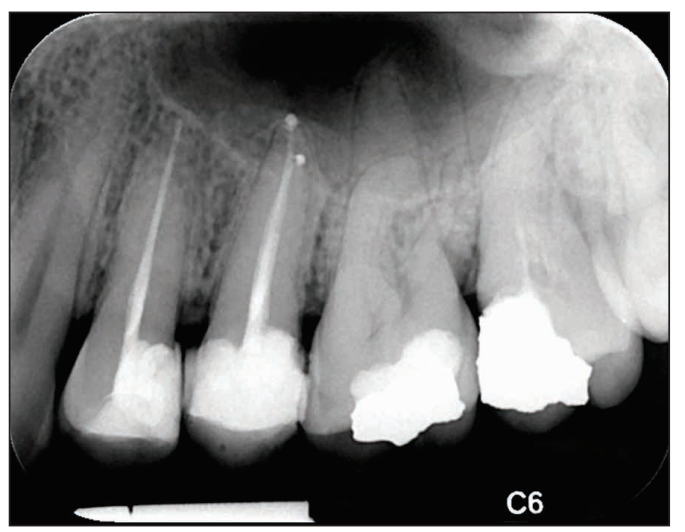

FIGURE 10. Apical puffs

\section{STRUCTURAL COMPLICATIONS IN ENDODONTICALLY TREATED TEETH}

Fracture of a cusp may occur at a different period after completion of endodontic treatment and conservative restoration of the tooth. The process 


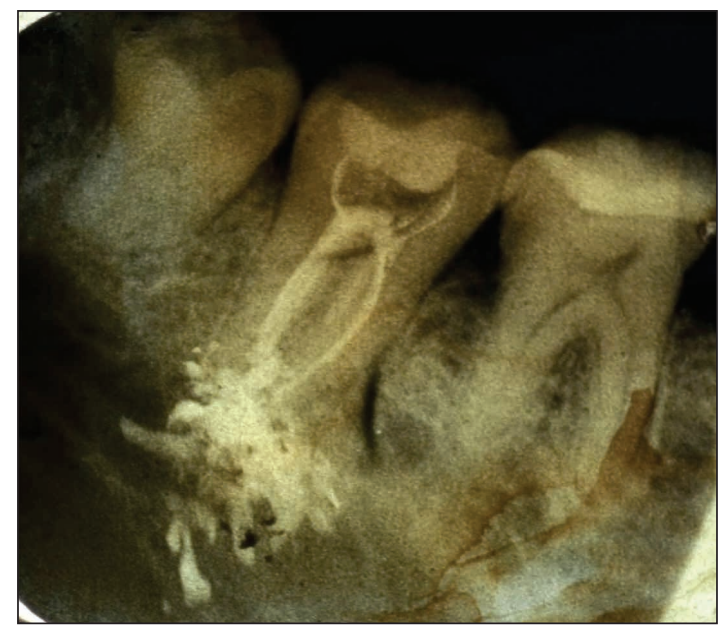

FIGURE 11. Large overfilling with sealer

is asymptomatic, the loose segment can be removed and a new adhesive restoration completed.

Fracture of a coronary wall. When the fracture does not affect the root canals, the restoration will be prosthetic, by covering with a crown.

Complete fracture of the tooth, affecting the root canals, usually vertical root fractures (VRF). In these cases, the tooth may not be restorable and it needs to be extracted (fig.12, 13).

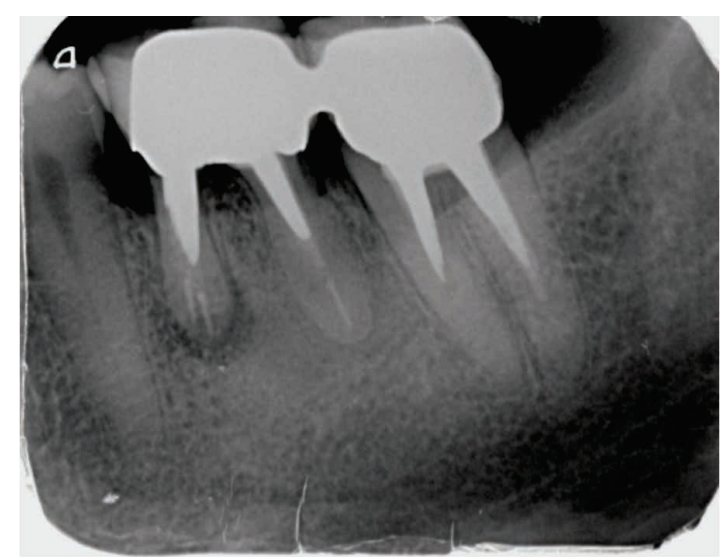

FIGURE 12. Extensive fracture between the mesial and distal roots

\section{REFERENCES}

1. Castellucci A. Access cavity and endodontic anatomy. In: Endodontics, vol. 1. Florence: II Tridente, 2007;244-253.

2. Arens DE, Gluskin $\mathrm{AH}$, Peters $\mathrm{Cl}$, Peters OA. Practical Lessons in Endodontic Treatment. Hanover Park: Quintessence Publ. Co. 2009;193-198

3. Bergmans L, Lambrechts P. Root canal instrumentation. In: Bergenholtz G, Horsted-Bindslev P, Reit C (eds.). Textbook of endodontology. 2nd ed. Oxford: Wiley-Blackwell Ltd. 2010;179-190.

4. Lumley P, Adams N, Tomson P. Practical Clinical Endodontics. Edinburgh: Churchill Livingstone Elsevier; 2006;35-54.

5. Plotino G, Grande NM, Falanga A, Di Giuseppe IL, Lamorgese V, Somma F. Dentine removal in the coronal portion of root canals following two preparation techniques. Int Endod J. 2007;40:852-858.

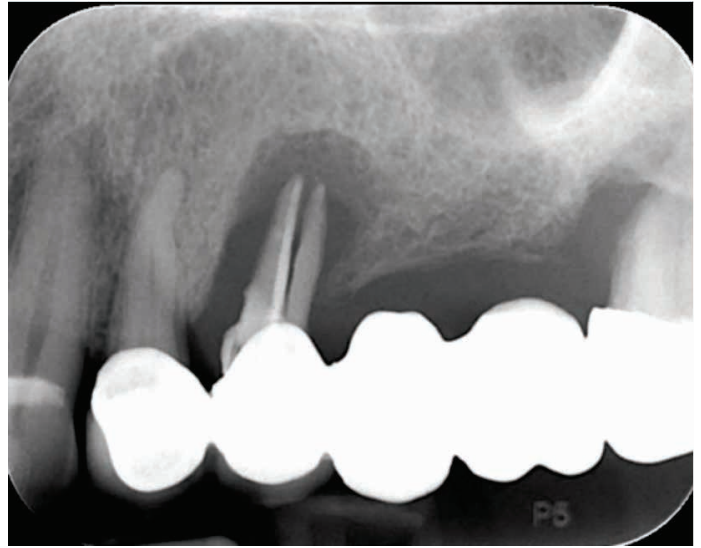

FIGURE 13. Vertical root fracture upper second premolar

\section{CONCLUSIONS}

The cleaning and shaping of the root canal system must be adapted to each canal individually, depending on its internal and external anatomical particularities - length, curvature, diameter per section. Disinfection and enlargement must take into account the amount of dental tissue left after the endodontic treatment. Thus, procedural errors, as a result of apical or lateral root canal over-instrumentation that destroy or decrease root strength, should be avoided. Infection is the most important factor influencing the prognosis of the treatment of iatrogenic errors and its outcome.

\section{Acknowledgement}

All authors have equal contributions to this article.

Conflict of interest: none declared Financial support: none declared
6. Scarlatescu SA. Gheorghiu IM, Sfeatcu R, Perlea P. Concepte actuale în lărgirea rotativă a canalelor radiculare. Ro J Stomatol. 2019;65(2):101-105.

7. f7. Gambarini G. Rational for the use of low-torque endodontic motors in root canal instrumentation. Endod Dent Traumatol. 2000;16:95-100.

8. Castellucci A. The Schilder technique of vertical compaction of warm gutta-percha. In: Endodontics, vol. 2. Florence: II Tridente, 2007; 653;667-687.

9. Beer R, Baumann MA, Kielbassa AM. Pocket atlas of endodontics. New York: Thieme Publ. 2006;166-169. 